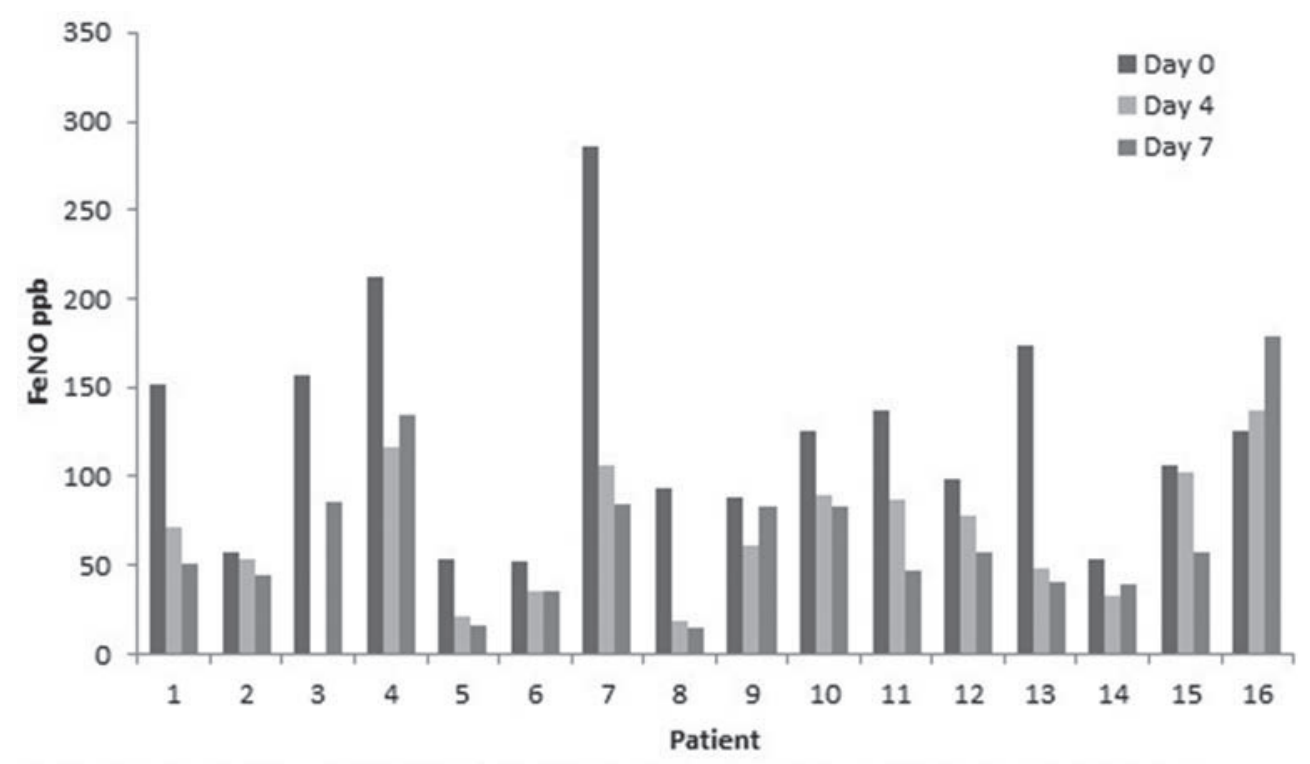

Abstract P12 Figure 1 FeNo values are depicted on the $Y$ axis and individual patients on the $X$ axis. Individual bars from left to right for a single subject depict FeNO values at days 0,4 and 7 respectively.

had $\geq 80 \%$ prescription pick-up rates checked with GP records. FeNO levels decreased from $121 \pm 63 \mathrm{ppb}$ to 71 $\pm 36 \mathrm{ppb}$ at day 4 and $66 \pm 43 \mathrm{ppb}$ at day 8 (p<0.01). 10 subjects had significant FeNO suppression by day 4 (figure 1). ACQ-7 score improved from $2.7 \pm 1.2$ to $1.7 \pm 1.1 \quad(\mathrm{p}<0.01)$. Eosinophil count $(0.65 \pm 0.51)$ and percent predicted FEV1 (79 $\pm 19)$ did not change significantly.

Conclusion Combining the use of once a day therapy with remote assessment using appropriate technology, FeNO suppression is a feasible objective test of adherence in the routine clinical setting. Despite appropriate refill collection rates over half of subjects were identified as non-adherent to inhaled therapy. Although these individuals did not commence biological therapy, improvements have been sustained. We recommend routine use of this assessment in severe asthma services.

\section{P13 BRONCHIAL THERMOPLASTY MAINTAINS A LONG-TERM REDUCTION IN PERIPHERAL BLOOD EOSINOPHILS IN SEVERE ASTHMA}

${ }^{1} \mathrm{~K}$ Hince, ${ }^{1} \mathrm{U}$ Holmes, ${ }^{1} \mathrm{G}$ McCumesky, ${ }^{2} \mathrm{D}$ Ryan, ${ }^{1} \mathrm{RM}$ Niven. ${ }^{1}$ University Hospital of South Manchester, Manchester, UK; ${ }^{2}$ Beaumont Hospital, Dublin, Ireland

\subsection{6/thoraxjnl-2017-210983.155}

Introduction Severe Asthma, characterised by persistent symptoms despite maximal medical therapy, represents $5 \%$ of asthma cases. Bronchial Thermoplasty (BT) is a novel therapy where radiofrequency thermal energy is applied to airways distal to the main-stem bronchi, permanently reducing airway smooth muscle mass. It is unknown whether treatment of smooth muscle hypertrophy impacts persistently upon systemic signs of allergic inflammation. Peripheral blood eosinophils (PBEs) are a marker of inflammation in asthma. We have previously shown that $\mathrm{BT}$ modifies signs of $\mathrm{Th}_{2}$ inflammation as measured by PBEs and therefore wanted to ascertain whether this persisted over time?

Method We reviewed the 13 consecutive Severe Asthma cases treated with BT who were included in the initial analysis. Serial PBEs measured up to 3 years post BT were compared to those in the year proceeding BT. Blood eosinophil levels taken peri-procedure were excluded from analysis due to escalated steroid therapy at this time.

Results Figure 1 demonstrates the absolute values of PBE for each patient during the 3 time periods (year before BT, Year immediately after BT and third year post BT). The group mean in year 3 has remained suppressed compared to baseline (year 3; 0.17 baseline $\mathrm{p}=0.0035$ ).

Conclusion Previous findings showed that Severe Asthma patients undergoing BT had a significant reduction in average peripheral blood eosinophil levels from baseline. The data shows overall the group of 13 patients continue to remain eosinophil supressed supporting the possibility of a long term eosinophil suppressive impact of BT.

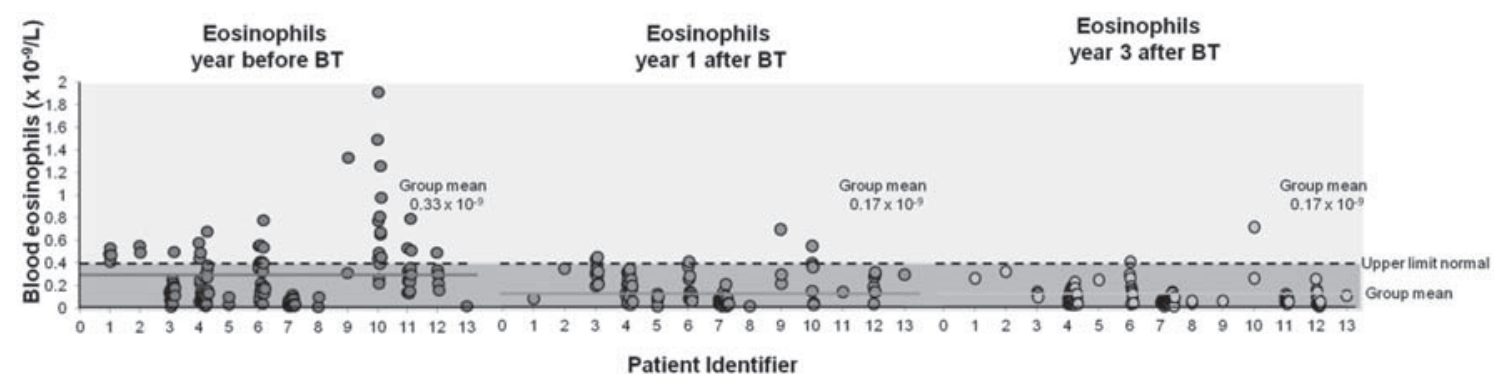

Abstract P13 Figure 1 Persistent suppression of eosinophils 3 years following bronchial thermoplasty (BT). 